\title{
In vitro susceptibility to methicillin, vancomycin and linezolid of staphylococci isolated from bloodstream infections in eastern Turkey
}

\author{
Tekin, Alicem ${ }^{1}$, Dal, Tuba ${ }^{1,}$ Deveci, Özcan ${ }^{2}$, Tekin, Recep ${ }^{2}$, Özcan, Nida $^{1}$, \\ Atmaca, Selahattin ${ }^{1}$, Dayan, Saim ${ }^{2}$ \\ ${ }^{1}$ Department of Microbiology and Clinical Microbiology, Medical Faculty, Dicle University, \\ Diyarbakir, Turkey. \\ ${ }^{2}$ Department of Clinical Microbiology and Infectious Diseases, Medical Faculty, Dicle University, \\ Diyarbakir, Turkey.
}

Submitted: December 16, 2012; Approved: December 13, 2013.

\begin{abstract}
Staphylococcus species are one of the major causes of bacterial bloodstream infections. Multiresistant staphylococci infections are major therapeutic problems. This study was aimed to detect methicillin, linezolid and vancomycin susceptibilities of Staphylococcus isolates. A total of 870 Staphylococcus strains isolated from blood cultures of hospitalized patients with BSI. Antimicrobial susceptibilities of methicillin, linezolid and vancomycin were detected according to the Clinical and Laboratory Standards Institute (CLSI). A total of 771 (88.6\%) isolates were coagulase-negative staphylococci (CoNS). 700 (80.5\%) isolates were methicillin-resistant (MR) and $170(19.5 \%)$ were methicillin-susceptible (MS). All the MS isolates were also susceptible to linezolid. However 15 $(1.7 \%)$ of MR strains were resistant to linezolid. The minimum inhibitory concentration range for the linezolid-resistant isolates by Etest was $6-32 \mu \mathrm{g} / \mathrm{mL}$. The difference between linezolid susceptibilities for MS and MR staphylococci was not quite statistically significant $(\mathrm{p}=0.052)$. There was no statistically significant difference between S. aureus and CoNS isolates for linezolid susceptibility. All of the isolates were susceptible to vancomycin. In conclusion, linezolid is currently an efficient option for the treatment of methicillin-resistant staphylococci infections.
\end{abstract}

Key words: Staphylococcus, methicillin, linezolid, vancomycin.

Bacterial bloodstream infections (BSI) are important problems worldwide. Staphylococcus species are one of the major causes of BSI, in both community- and hospitalacquired diseases. Staphylococci are widespread in nature, also they are commensal microorganisms located on the surface of the human skin and mucous membranes (Bannerman, 2003; Winn et al., 2006; Yok-Ai Que and Moreillon, 2010). Staphylococcus aureus and coagulase-negative staphylococci (CoNS), mainly Staphylococcus epidermidis, are the most common bacteria isolated from blood (Bannerman, 2003; Natoli et al., 2009; Winn et al., 2006). In addition, Yok-Ai Que and Moreillon (2010) stated that CoNS may adhere to medical devices and hospital environmental surfaces by forming bio-films. These bacteria can easily spread from person to person through patient fluids, contaminated medical devices and on the hands of health-care workers (Bannerman, 2003; Yok-Ai Que and Moreillon, 2010). The isolation rate of $S$. aureus from BSIs has universally increased. Wisplinghoff et al. (2004) reported that CoNS and S. aureus were responsible for approximately $30 \%$ and $20 \%$ of the nosocomial BSIs, respectively.

In recent years, infections caused by multi-resistant staphylococci have become one of the major therapeutic problems (Deveci et al., 2011). The emergence of these multidrug resistant isolates is often attributed to long term usage of indwelling central venous catheters, administration of parenteral nutrition, long term usage of broadspectrum antibiotics and increasing number of immunocompromised patients (Winn et al., 2006; Yok-Ai Que and 
Moreillon, 2010). These problematic pathogens have acquired resistance to methicillin and some of which to many non-beta-lactam antibiotics (Boyle-Vavra and Daum, 2007; Niemeyer et al., 1996; Winn et al., 2006). Methicillin resistance in staphylococci is caused by expression of the penicillin-binding protein 2a (PBP2a) encoded by the mecA gene which is located in the staphylococcal cassette chromosome mec ( $\mathrm{SCCmec}$ ) and can be transmitted via horizontal transfer between Staphylococcus species (Boyle-Vavra and Daum, 2007; Niemeyer et al., 1996; Yok-Ai Que and Moreillon, 2010). Currently, over $80 \%$ of clinical Staphylococcus isolates throughout the world are resistant to penicillin due to $\beta$-lactamase production (Winn et al., 2006). Until recently, glycopeptides such as vancomycin were the first choice drug to treat infections caused by methicillin-resistant staphylococci (Yok-Ai Que and Moreillon, 2010). However, vancomycin-resistant Staphylococcus isolates have been detected in various countries (Centers for Disease Control and Prevention (CDC), 1997, 1999, 2002, 2004; Gemmell et al., 2001; Hiramatsu et al., 1997; Palazzo et al., 2005).

Linezolid was introduced by Ford et al. (1997) as a member of the oxazolidinone class of antibiotics. This antibiotic demonstrates potent antimicrobial activity against most multi-resistant Gram-positive microorganisms, including methicillin-resistant coagulase-negative staphylococci (MRCoNS), methicillin-resistant S. aureus (MRSA), multidrug-resistant (MDR) Streptococcus pneumoniae and vancomycin-resistant enterococci (VRE) (Ford et al., 1997). Linezolid inhibits bacterial protein syntheses by binding to the domain $\mathrm{V}$ region of $23 \mathrm{~S}$ rRNA at an early step, and prevents the formation of the $\mathrm{N}$-formylmethionyl-tRNA-mRNA-70S ribosomal tertiary complex. Mutations in the central loop of the domain $\mathrm{V}$ region are the most frequent causes of linezolid resistance (Khan et al., 2012; Kloss et al., 1999).

This study presented here aimed to detect methicillin, linezolid and vancomycin susceptibilities for CoNS and $S$. aureus isolates collected from blood cultures in Dicle University Hospital, Diyarbakir-Turkey.

A total of 870 staphylococcal isolates collected from blood cultures of hospitalized patients in Dicle University Hospital between January 2007 and August 2011 were included retrospectively in this study. Dicle University Hospital is a tertiary care center with capacity of 1400 beds. Among 870 staphylococcal isolates included in this study, 442 were collected from adult patients (ranged 17-65 years), 237 from pediatric patients ( $\leq 16$ years) and 191 from newborn babies admitted into the neonatal intensive care unit (NICU). Only isolates from clinically significant BSI (one isolate per patient) were included in this study. Clinically significant BSI can be diagnosed when two or more of the following criteria are present with positive blood cultures taken from both arms: -body temperature $\leq 36{ }^{\circ} \mathrm{C}$ or $\geq 38^{\circ} \mathrm{C}$; -heart rate $>90$ beats $/$ min; -respiratory rate $>20$ breaths $/$ min or an arterial partial pressure of carbon dioxide $\left(\mathrm{PaCO}_{2}\right)<4.3 \mathrm{kPa}(32 \mathrm{mmHg})$; -leukocytes $<4,000$ cells $/ \mathrm{mm}^{3}\left(4 \times 10^{9}\right.$ cells $\left./ \mathrm{L}\right)$ or $>12,000$ cells $/ \mathrm{mm}^{3}$ $\left(12 \times 10^{9}\right.$ cells $\left./ \mathrm{L}\right)$ or $>10 \%$ immature neutrophils (band forms) (American college of chest physicians / society of critical care medicine consensus conference, 1992). Blood culture bottles were incubated in Bactec ${ }^{\mathrm{TM}}$ BD 9120 and 9240 (Becton Dickinson, MD, USA) automated blood culture systems at $37^{\circ} \mathrm{C}$ for $7-10$ days. After growth, the culture was inoculated onto 5\% sheep blood agar (Oxoid Ltd., Basingstoke, UK) and the plate incubated at $35 \pm 2{ }^{\circ} \mathrm{C}$ for 18-24 h. Isolate identification was performed by routine methods of Gram staining, catalase activity, slide and tube coagulase tests, DNAse test, and also BD Phoenix ${ }^{\mathrm{TM}} 100$ (Becton Dickinson, MD, USA) the fully automated microbiology system by using the manufacturers' protocol.

Antimicrobial susceptibility testing for methicillin and linezolid were performed by Kirby-Bauer's disk diffusion method and BD Phoenix ${ }^{\mathrm{TM}} 100$ the fully automated microbiology system by using the manufacturers' protocol according to the recommendations of Clinical and Laboratory Standards Institute (CLSI) (2009). Methicillin susceptibility was investigated with $1 \mu \mathrm{g}$ oxacillin and $30 \mu \mathrm{g}$ cefoxitin disk (Oxoid Ltd., Basingstoke, UK). Linezolid susceptibility was tested using $30 \mu \mathrm{g}$ linezolid disk (Oxoid Ltd., Basingstoke, UK) and linezolid resistance was confirmed by Etest strips (bioMerieux SA, Marcy l'Etoile, France) (Abb, 2002; CLSI, 2009). In addition, vancomycin susceptibility was also investigated using Etest strips and the fully automated microbiology system according to the CLSI breakpoints (2009) For disk diffusion method and Etest strips, a $0.5 \mathrm{McF}$ arland standard suspension was inoculated onto Mueller-Hinton agar (Merck KGaA, Darmstadt, Germany) plates as described by CLSI (2009). MHA plates were incubated at $35 \pm 2{ }^{\circ} \mathrm{C}$ for 24 hours. Inhibition zone diameter was measured at 24 hours in transmitted light for linezolid.

For methicillin susceptibility, in accordance with CLSI guidelines, inhibition zone diameter for oxacillin $\geq 13 \mathrm{~mm}$ was considered as susceptible, $11-12 \mathrm{~mm}$ as intermediate, $\leq 10 \mathrm{~mm}$ as resistant; for cefoxitin $\geq 22 \mathrm{~mm}$ was considered as susceptible, $\leq 21 \mathrm{~mm}$ as resistant for $S$. aureus and $S$. lugdunensis, $\geq 25 \mathrm{~mm}$ was considered as susceptible, $\leq 24 \mathrm{~mm}$ as resistant for the other CoNS except $S$. lugdunensis. Inhibition zone diameter for linezolid $\geq 21 \mathrm{~mm}$ was considered as susceptible, $<21 \mathrm{~mm}$ as resistant. MIC value for linezolid $\leq 4 \mu \mathrm{g} / \mathrm{mL}$ was considered as susceptible, $>4 \mu \mathrm{g} / \mathrm{mL}$ as resistant. According to the MIC interpretive standard of CLSI, MIC value for vancomycin $\geq 2 \mu \mathrm{g} / \mathrm{mL}$ was considered as susceptible, $4-8 \mu \mathrm{g} / \mathrm{mL}$ as intermediate, $\geq 16 \mu \mathrm{g} / \mathrm{mL}$ as resistant.

S. aureus ATCC 25923 was used for quality control in the fully automated microbiology system, Etest strips and disk diffusion method. Data were analyzed by Epi 
Info $^{\mathrm{TM}}$ 7-Community Edition (Centers for Diseases Control and Prevention, Atlanta, GA, USA) statistical package program. Statistical evaluation between the groups of methicillin-susceptible and -resistant isolates for linezolid susceptibility was performed with the Fisher's exact test. Also, statistical evaluation between $S$. aureus and CoNS isolates for linezolid susceptibility was analyzed with the chisquare test. The $\mathrm{p}$-value of $<0.05$ was selected for statistical significance.

In the present study, $771(88.6 \%)$ of 870 isolates were coagulase-negative staphylococci (CoNS), and 99 (11.4\%) $S$. aureus. According to the data from the antimicrobial susceptibility testing, $700(80.5 \%)$ isolates were methicillinresistant staphylococci (MRS), and 170 (19.5\%) methicillin-susceptible staphylococci (MSS). All the MSS isolates were also susceptible to linezolid. However 15 (1.7\%) of MRS strains were resistant to linezolid. The MIC range for the linezolid-resistant isolates using Etest was 6-32 $\mu \mathrm{g} / \mathrm{mL}$. In addition, the MIC value for all the linezolid-resistant isolates using the fully automated microbiology system was recorded as $>4 \mu \mathrm{g} / \mathrm{mL}$ uniformly. The difference between linezolid susceptibilities for MSS and MRS was not quite statistically significant $(\mathrm{p}=0.052)$. Among 15 methicillin- and linezolid-resistant staphylococcal isolates, only one was $S$. aureus and the others were CoNS $(\leq)$. There was no statistically significant difference between $S$. aureus and CoNS isolates for linezolid susceptibility. All staphylococci tested were susceptible to vancomycin according to the both Etest and the fully automated microbiology system. The MIC value for all the vancomycin susceptible isolates using the both Etest and the fully automated microbiology system was recorded as $\leq 2 \mu \mathrm{g} / \mathrm{mL}$ uniformly.

CoNS and $S$. aureus are frequent causes of bacteremia and sepsis, in both community and hospitalized patients (Natoli et al., 2009; Winn et al., 2006). In recent years, due to the increasing antimicrobial resistance, treatment of staphylococcal infections has become more challenging. Currently, over $80 \%$ of clinical staphylococcal isolates are resistant to penicillin worldwide. In addition over $60 \%$ of nosocomial $S$. aureus and CoNS isolates are resistant to methicillin in some Asian countries including China and Turkey. Lin et al. (2008) stated methicillinresistant staphylococci as also cross-resistant to other $\beta$-lactams and to a wide range of other antibiotics (22). In our study $80.5 \%$ of total 870 isolates were MRS. Therefore, beta-lactams are inefficacious agents to treat staphylococcal infections in our hospital.

Until recently, most Staphylococcus isolates were susceptible to glycopeptides such as vancomycin. The first cases of infections with glycopeptides-resistant $S$. haemolyticus were reported in 1986 and the first VISA isolate was described in Japan (Hiramatsu et al., 1997). Shortly after, Staphylococcus isolates with reduced susceptibility to vancomycin, and with intermediate and full resis- tance to this drug were also reported in many countries (Bataineh, 2006; Howe, 1998; Palazzo, 2005). At the beginning of the second millennium, eight cases of vancomycin-intermediate $S$. aureus (VISA) and one of vancomycin-resistant $S$. aureus (VRSA) (MIC value for vancomycin $\geq 32 \mu \mathrm{g} / \mathrm{mL}$ ) were documented in the United States (CDC, 2002). Palazzo et al. (2005) reported vancomycinresistant staphylococcal isolates in healthy carriers that have not received antibiotic treatment.

In Turkey, some isolates showing reduced susceptibility or intermediate resistance for vancomycin had also been reported. Torun et al. (2005) reported two heterogeneously vancomycin-intermediate clinical isolates of methicillin-susceptible and methicillin-resistant $S$. aureus. Sancak et al. (2005) have also documented a MRSA isolate displaying heterogeneous resistance to vancomycin in a Turkish university hospital. Despite that, there was no report of vancomycin full resistance among staphylococcal isolates in Turkey. In the present study, all staphylococci analyzed were susceptible to vancomycin. Indeed, VISA isolates could not be detected by the susceptibility testing methods used. The fact that we have used the fully automated microbiology system could have influenced the VISA negative detection. However, we have also included the Etest for determining the MIC value for vancomycin. MIC determinations by agar or broth dilution, in addition to Etest using a $0.5 \mathrm{McF}$ arland turbidity standard, have been recommended for detecting VISA isolates (Sader et al., 2009). The only observation is that in this case susceptibility breakpoint should be adjusted to $\leq 1 \mu \mathrm{g} / \mathrm{mL}$. Sader et al. (2009) have suggested that the susceptibility breakpoint be modified to $\leq 1 \mu \mathrm{g} / \mathrm{mL}$.

In the present study we found that only $1.7 \%$ of total 870 staphylococcal isolates was resistant to linezolid according to the both disk diffusion method and the fully automated microbiology system. Linezolid resistance was confirmed by using Etest strips in this study. Many researchers have accepted that the Etest method is credible method to detect the MIC value (Bell et al., 2003; CLSI, 2009; Kaya et al., 2009; Thool et al., 2012). But CLSI (2009) recommended the confirmation of resistance to linezolid by using agar or broth dilutions. In addition, Gemmell et al. (2001) said that the mean Etest MIC was approximately one two-fold dilution lower than the mean microdilution MIC. Also Tenover et al. (2007) reported that further studies of the agar-based methods (disk diffusion method and Etest) are needed to better define the optimal endpoints for interpreting results of testing for linezolid against staphylococci and enterococci. Arias et al. (2008) stated that disk diffusion susceptibility tests or Etest might not detect $c f r$-mediated linezolid resistance when standard procedures are used and that a longer time of incubation may be needed.

Thus, linezolid is still an effective agent for staphylococcal isolates obtained from blood culture in our hospital. 
Table 1 - Bacterial species and hospital setting for methicillin-linezolid resistant staphylococcal isolates.

\begin{tabular}{lcc}
\hline Isolate no & Species & Inpatient Clinics \\
\hline DUH1 & S. kloosii & Plastic Surgery \\
DUH2 & S. aureus & Nephrology \\
DUH3 & S. hyicus & Pediatric Infectious Diseases \\
DUH4 & S. cohnii & Oncology \\
DUH5 & S. cohnii & Chest Diseases \\
DUH6 & S. kloosii & Neonatal Intensive Care Unit \\
DUH7 & S. cohnii & Cardiology \\
DUH8 & S. kloosii & Neonatal Intensive Care Unit \\
DUH9 & S. schleiferi & Neonatal Intensive Care Unit \\
DUH10 & S. kloosii & Pediatric Infectious Diseases \\
DUH11 & S. capitis & Neonatal Intensive Care Unit \\
DUH12 & S. kloosii & Neonatal Intensive Care Unit \\
DUH13 & S. capitis & Endocrinology \\
DUH14 & S. cohnii & Adult Infectious Disease \\
DUH15 & S. hominis & Hematology \\
\hline
\end{tabular}

Data from LEADER surveillance program in USA, from linezolid program (ZyvoxR Annual Appraisal of Potency and Spectrum; ZAAPS) in European countries and from many studies in Spain and in our country found that linezolid sensitivity rates in staphylococci were approximately 99\% (Jones et al., 2007; Ross et al., 2011). It is important to remark that five linezolid-resistant $S$. kloosii isolates were obtained from hospitalized patients in the plastic surgery (one isolate), pediatrics clinic (one isolate) and neonatal intensive care unit (NICU; three isolates). This result seems to indicate the occurrence of $S$. kloosii small outbreak in the NICU of the studied hospital. Recently, Peer et al. (2011) reported a case of sepsis with an intracranial bleed in a 60-year-old male from whom linezolid-resistant $S$. kloosii was repeatedly isolated from blood cultures, demonstrating the potential of this staphylococcal species to cause BSI in immunocompromised host. S. epidermidis was not detected among the linezolid-resistant isolates in the present study. However, it is possible that the fully automated identification system used may have failed to detect some of the resistant isolates.

Due to the emergence of vancomycin-intermediate and -resistant isolates, poor tissue penetration, and the fact that vancomycin must be given intravenously, new therapeutic options are necessary for the treatment of staphylococcal infections. Our data showed that, in our hospital, linezolid can be a therapeutic option for treatment of infections cause by both MRCoNS and MRSA. Linezolid is a well-tolerated agent currently available in oral and intravenous forms. The oral form is $100 \%$ bio-available and the earlier switching from intravenous to oral therapy is possi- ble (Weigelt et al., 2005). In a cohort study of Lodise et al. (2008), the incidence of nephrotoxicity with higher daily ( $\geq 4 \mathrm{~g}$ /day) and lower daily doses of vancomycin $<4 \mathrm{~g} /$ day) were compared with nephrotoxicity of linezolid. According to this study, nephrotoxicity was significantly higher in the high-dose vancomycin group (34.6\%) when compared with the low-dose vancomycin group $(10.9 \%)$ or with the linezolid group (6.7\%).

In conclusion, the treatment of methicillin-resistant staphylococcal infections has been a growing problem in both hospital- and community-acquired diseases. For this reason, alternative antimicrobial agents with antistaphylococcal activity are needed. The results presented here are in line with data of previous national and international investigations suggesting that linezolid is an efficient option for the treatment of a number of infections caused by methicillin-resistant staphylococci (Lin et al., 2008; Weigelt et al., 2005; Zorgani et al., 2012). Finally, due to the increasing antimicrobial resistance among staphylococcal isolates, resistance rates should be reported periodically.

\section{References}

Abb J (2002) In vitro activity of linezolid, quinupristin-dalfopristin, vancomycin, teicoplanin, moxifloxacin and mupirocin against methicillin-resistant Staphylococcus aureus: comparative evaluation by the E test and a broth microdilution method. Diagn Microbiol Infect Dis 43:319-321.

American college of chest physicians / society of critical care medicine consensus conference (1992) Definitions for sepsis and organ failure and guidelines for the use of innovative therapies in sepsis. Crit Care Med 20:864-74.

Arias CA, Vallejo M, Reyes J, Panesso D, Moreno J, Castaneda E, Villegas MV, Murray BE, Quinn JP (2008) Clinical and microbiological aspects of linezolid resistance mediated by the cfr gene encoding a $23 \mathrm{~S}$ rRNA methyltransferase. J Clin Microbiol 46:892-896.

Bannerman TL (2003) Staphylococcus, Micrococcus, and other catalase-positive cocci that grow aerobically. In: Murray PR, Baron EJ, Jorgensen JH, Pfaller MA, Yolken RH (eds) Manual of Clinical Microbiology. 8th ed. ASM Press, Washington, DC, p. 384-404.

Bataineh AB (2006) Resistance of Staphylococcus aureus to Vancomycin in Zarqa, Jordan. Pak J Med Sci 22:144-148.

Bell JM, Turnidge JD, Ballow CH, Jones RN, the ZAPS Regional Participants (2003) Multicentre evaluation of the in vitro activity of linezolid in the Western Pacific. J Antimicrob Chemother 51:339-345.

Boyle-Vavra S, Daum RS (2007) Community-acquired methicillin-resistant Staphylococcus aureus: the role of PantonValentine leukocidin. Laboratory Investigation 87:3-9.

Centers for Disease Control and Prevention (1997) Staphylococcus aureus with reduced susceptibility to vancomycinUnited States, 1997. MMWR Morb Mortal Wkly Rep 46:765-766.

Centers for Disease Control and Prevention (1999) Staphylococcus aureus with reduced susceptibility to vancomycin- 
Illinois, 1999. MMWR Morb Mortal Wkly Rep 48:11651167.

Centers for Disease Control and Prevention (2002) Staphylococcus aureus resistant to vancomycin-United States. Morb Mortal Wkly Rep 51:565-567.

Centers for Disease Control and Prevention (2004) Vancomycinresistant Staphylococcus aureus. MMWR Morb Mortal Wkly Rep 53:322-323.

Clinical and Laboratory Standarts Institute (2009) Performance standarts for antimicrobial susceptibility testing: $19^{\text {th }}$ informational supplement. CLSI Document M100-S19, Wayne.

Deveci O, Kilic D, Kaygusuz S, Duruyurek N, Karabicak C, Agalar C, Tekin A (2011) Evaluation of resistance to fusidic acid in Staphylococci isolates. J Microbiol Infect Dis $1: 22-25$.

Ford CW, Hamel JC, Stapert D, Moerman JK, Hutchinson DK, Barbachyn MR, et al. (1997) Oxazolidinones: New antibacterial agents. Trends in Microbiology 5:196-200.

Gemmell CG et al. (2001) Susceptibility of a variety of clinical isolates to linezolid: a European inter-country comparison. J Antimicrob Chemother 48:47-52.

Hiramatsu K, Hanaki H, Ino T, Yabuta K, Oguri T, Tenover FC (1997) Methicillin resistant Staphylococcus aureus clinical strain with reduced vancomycin susceptibility. J Antimicrob Chemother 40:135-136.

Howe RA, Bowker KE, Walsh TR, Feest TG, MacGowan AP (1998) Vancomycin-resistant Staphylococcus aureus. Lancet 351:602.

Jones RN, Fritsche TR, Sader HS, Ross JE (2007) LEADER surveillance program results for 2006: an activity and spectrum analysis of linezolid using clinical isolates from the United States (50 medical centers). Diagn Microbiol Infect Dis 59:309-317.

Kaya O, Akcam FZ, Temel EN (2008) In vitro activities of linezolid and tigecycline against methicillin-resistant Staphylococcus aureus strains. Microb Drug Resist 14:151-153.

Khan MF, Neral A, Yadav VC, Khan FA, Ahmed S (2012) Emergence of linezolid resistant Staphylococcus aureus in Bastar tribal region, India. J Microbiol Infect Dis 2:127-128.

Kloss P, Xiong L, Shinabarger DL, Mankin AS (1999) Resistance mutations in 23S rRNA identify the site of action of the protein synthesis inhibitor linezolid in the ribosomal peptidyl transferase center. J Mol Biol 294:93-101.

Lin DF, Zhang YY, Wu JF, Wang F, Zheng JC, Miao JZ (2008) Linezolid for the treatment of infections caused by Grampositive pathogens in China. Int $\mathrm{J}$ Antimicrob Agents 32:241-249.

Lodise TP, Lomaestro B, Graves J, Drusano GL (2008) Larger vancomycin doses (at least four grams per day) are associated with an increased incidence of nephrotoxicity. Antimicrob Agents Chemother 52:1330-1336.

Natoli S, Fontana C, Favaro M, Bergamini A, Testore GP, Minelli S (2009) Characterization of coagulase-negative staphylococcal isolates from blood with reduced susceptibility to glycopeptides and therapeutic options. BMC Infect Dis 9:83.

Niemeyer DM, Pucci MJ, Thanassi JA, Sharma VK, Archer GL (1996) Role of mecA transcriptional regulation in the phenotypic expression of methicillin resistance in Staphylococcus aureus. J Bacteriol 178:5464-5471.
Palazzo ICV, Araujo MLC, Darini ALC (2005) First report of vancomycin-resistant staphylococci isolated from healthy carriers in Brazil. J Clin Microbiol 43:179-185.

Peer M, Nasir R, Kakru D, Fomda B, Bashir G, Sheikh I (2011) Sepsis due to linezolid resistant Staphylococcus cohnii and Staphylococcus kloosii: First reports of linezolid resistance in coagulase negative staphylococci from India. Indian J Med Microbiol 29:60.

Ross JE, Farrell DJ, Mendes RE, Sader HS, Jones RN (2011) Eight year (2002-2009) summary of the linezolid (ZyvoxR Annual Appraisal of Potency and Spectrum; ZAAPS) program in European countries. J Chemother 23:71-76.

Sader HS, Rhomberg PR, Jones RN (2009) Nine-hospital study comparing broth microdilution and Etest method results for vancomycin and daptomycin against methicillin-resistant Staphylococcus aureus. Antimicrob Agents Chemother 53:3162-3165.

Sancak B, Ercis S, Menemenlioglu D, Colakoglu S, Hascelik G (2005) Methicillin-resistant Staphylococcus aureus heterogeneously resistant to vancomycin in a Turkish university hospital. J Antimicrob Chemother 56:519-523.

Tenover FC, Williams PP, Stocker S, Thompson A, Clark LA, Limbago B, Carey RB, Poppe SM, Shinabarger D, McGowan JE (2007) Accuracy of six antimicrobial susceptibility methods for testing linezolid against Staphylococci and Enterococci. J Clin Microbiol 45:2917-2922.

Thool VU, Bhoosreddy GL, Wadher BJ (2012) Detection of resistance to linezolid in Staphylococcus aureus infecting orthopedic patients. IndianJ Pathol Microbiol 55:361-364.

Torun MM, Bahar H, Demirci M, Altas K, Bagdatli Y, Kocazeybek B (2005) Two heterogeneously vancomycinintermediate clinical isolates of methicillin-sensitive and methicillin-resistant Staphylococcus aureus in a Turkish university hospital: brief report of a surveillance study. Int J Antimicrob Agents 26:508-510.

Weigelt J, Itani K, Stevens D, Lau W, Dryden M, Knirsch C (2005) Linezolid $v s$. vancomycin in treatment of complicated skin and soft tissue infections. Antimicrob Agents Chemother 49:2260-2266.

Winn WJ, Allen S, Janda W, Koneman E, Procop G, Schreckenberger P, Woods G (eds) (2006) Staphylococci and related Gram-positive cocci. Color Atlas and Textbook of Diagnostic Microbiology. 6th ed. Philadelphia, Lippincott Williams and Wilkins, p 623-671.

Wisplinghoff H, Bischoff T, Tallent SM, Seifert H, Wenzel RP, Edmond MB (2004) Nosocomial bloodstream infections in US hospitals: analysis of 24,179 cases from a prospective nationwide surveillance study. Clin Infect Dis 39:309-317.

Yok-Ai Que, Moreillon P (2010) Staphylococcus aureus (Including Staphylococcal Toxic Shock). In: Mandell GL, Bennett JE, Dolin R (eds). Mandell, Dauglas, and Bennett's Principles and Practice of Infectious Diseases. 7th ed. Philadelphia: Churchill Livingstone Elsevier, p 2543-2578.

Zorgani A, Elahmer O, Ziglam H, Ghenghesh KS (2012) In-vitro activity of tigecycline against methicillin-resistant Staphylococcus aureus isolated from wounds of burn patients in Tripoli-Libya. Journal of Microbiology and Infectious Diseases 2:109-112.

All the content of the journal, except where otherwise noted, is licensed under a Creative Commons License CC BY-NC. 